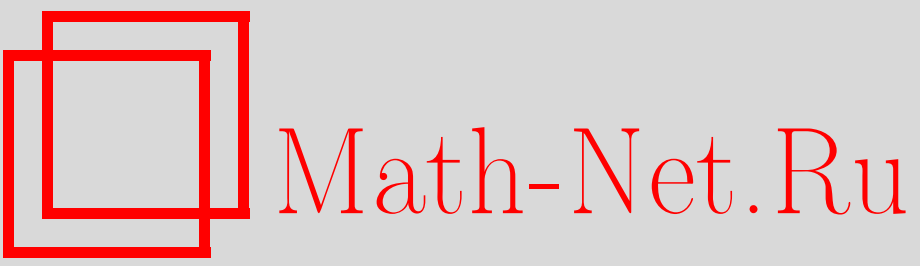

М. А. Всемирнов, М. Г. Ржевский, Верхняя оценка контактного числа в размерности 9, УМH, 2002, том 57, выпуск 5, 149-150

DOI: https://doi.org/10.4213/rm559

Использование Общероссийского математического портала Math-Net.Ru подразумевает, что вы прочитали и согласны с пользовательским соглашением

http://www . mathnet.ru/rus/agreement

Параметры загрузки:

IP : 54.84 .234 .179

26 апреля 2023 г., 13:57:08 


\title{
ВЕРХНЯЯ ОЦЕНКА КОНТАКТНОГО ЧИСЛА В РАЗМЕРНОСТИ 9
}

\author{
М. А. ВСемиРнов, М. Г. РЖЕВСКИЙ
}

Контактным числом $\tau_{d}$ евклидова пространства $\mathbb{R}^{d}$ называют максимальное число шаров единичного радиуса с непересекающимися внутренностями, касающихся одного такого же шара. Эквивалентным образом, $\tau_{d}$ есть максимальная мощность 1/2-сферического кода, т.е. такого набора точек на единичной сфере $S^{d-1}$, в котором для любых двух различных точек $x$ и $y$ имеем $(x, y) \leqslant$ $1 / 2$. Здесь $(x, y)$ - обычное скалярное произведение в $\mathbb{R}^{d}$. Задача нахождения точного значения $\tau_{d}$ очень сложна. В настояший момент она решена лишь для размерностей $d=2,3,8,24$; в остальных случаях известны лишь двусторонние оценки на размер контактного числа, см. [1], [2]. Более того, даже незначительное улучшение этих оценок требует привлечения болшших усилий. Так, в [3] получены оценки $\tau_{19} \leqslant 25900, \tau_{21} \leqslant 56851$ и $\tau_{23} \leqslant 128095$, которые лишь на 1 уменьшают соответствующие оценки из [1], [2]. В осталшных размерностях $d \leqslant 24$ лучшими на настоящий момент оставались оценки из [1], [2]. Например, в [2] показано, что $\tau_{9} \leqslant 380$. С другой стороны, известно (см. [1]), что $\tau_{9} \geqslant 306$.

В настоящей заметке мы устанавливаем следующий резултат.

Teopema 1. $\tau_{9} \leqslant 379$.

Общая схема доказательства следует работам [1], [2], [4] и основана на сведении к задаче линейного программирования. При этом использются дополнительные неравенства, возникающие из естественных геометрических ограничений.

Пусть $C-1 / 2$-сферический код в $\mathbb{R}^{d}$. Через $\alpha(t)$ обозначим функцию распределения кода $C$, т.e.

$$
\alpha(t)=\frac{\#\left\{\langle x, y\rangle \in C^{2}:(x, y)=t\right\}}{\# C} .
$$

Пусть $\Phi_{k, d}(t)=P_{k}^{((d-3) / 2,(d-3) / 2)}(t)-($ ульрасферические) многочлены Якоби, нормализованные условием $P_{k}^{((d-3) / 2,(d-3) / 2)}(1)=1$. Хорошо известно (см., например, [1], [4]), что имеют место следующие неравенства:

$$
\sum_{-1 \leqslant t \leqslant 1 / 2} \alpha(t) \Phi_{k, d}(t) \geqslant-1 \text { для всех } k=1,2, \ldots
$$

Пусть $x_{1}, \ldots, x_{m+1}, z \in C$. Так как $\left(x_{i}, x_{j}\right) \leqslant 1 / 2$ при $i \neq j$ и $\left(x_{i}, x_{i}\right)=(z, z)=1$, то

$$
m+1+\frac{m(m+1)}{2} \geqslant \sum_{i, j}\left(x_{i}, x_{j}\right)=\left(\sum_{i=1}^{m+1} x_{i}, \sum_{i=1}^{m+1} x_{i}\right) \geqslant\left(\sum_{i=1}^{m+1} x_{i}, z\right)^{2}=\left(\sum_{i=1}^{m+1}\left(x_{i}, z\right)\right)^{2} .
$$

В частности, если $\left(x_{i}, z\right)<0$ для всех $i=1, \ldots, m+1$, то

$$
\max _{i=1, \ldots, m+1}\left(x_{i}, z\right) \geqslant t_{m}, \text { где } t_{m}=-\sqrt{\frac{m+2}{2 m+2}} .
$$

Таким образом, для всех $m=1,2, \ldots$ имеют место следующие неравенства:

$$
\sum_{-1 \leqslant t<t_{m}} \alpha(t) \leqslant m
$$

Работа выполнена при частичной поддержке Cariplo Foundation (Italy) и гранта 0016 -го конкурса-экспертизы научных проектов молодых ученых РАН. 
Пусть, кроме того, $r_{2}=-1 / 2-1 / \sqrt{6}$. Нетрудно проверить, что если $x, z \in C$ и $(x, z) \leqslant r_{2}$, то не существует такого вектора $y \in C(y \neq x)$, что $(y, z)<t_{2}$. Это дает неравенство

$$
2 \sum_{-1 \leqslant t \leqslant r_{2}} \alpha(t)+\sum_{r_{2}<t<t_{2}} \alpha(t) \leqslant 2
$$

Зафиксируем $N$ и рассмотрим задачу нахождения супремума (на самом деле, максимума) $\tau_{d}^{*}$ функционала $1+\sum_{t \leqslant 1 / 2} \alpha(t)$ при условиях $\alpha(t) \geqslant 0$ для всех $t \in[-1,1 / 2]$ и неравенствах (1) для $k=1, \ldots, N,(2)$ для $m=1$ и (3). Ясно, что $\tau_{d} \leqslant \tau_{d}^{*}$. Перейдем к двойственной задаче. Учитывая, что $\tau_{d}^{*}$ не превосходит любого допустимого решения двойственной задачи, получаем следующий результат.

ТЕОРема 2. Пусть числа $f_{1}, \ldots, f_{N+2}$ удовлетворяют неравенствам

$$
\begin{aligned}
& f_{i} \geqslant 0 \text { для всех } i=1, \ldots N+2, \\
& \sum_{k=1}^{N} f_{k} \Phi_{k, d}(t)-f_{N+1}-2 f_{N+2} \leqslant-1 \text { для всех } t \in[-1,-1 / 2-1 / \sqrt{6}], \\
& \sum_{k=1}^{N} f_{k} \Phi_{k, d}(t)-f_{N+1}-f_{N+2} \leqslant-1 \text { для всех } t \in(-1 / 2-1 / \sqrt{6},-\sqrt{3} / 2), \\
& \sum_{k=1}^{N} f_{k} \Phi_{k, d}(t)-f_{N+2} \leqslant-1 \text { для всех } t \in[-\sqrt{3} / 2,-\sqrt{2 / 3}), \\
& \sum_{k=1}^{N} f_{k} \Phi_{k, d}(t) \leqslant-1 \text { для всех } t \in[-\sqrt{2 / 3}, 1 / 2] .
\end{aligned}
$$

Тогда

$$
\tau_{d} \leqslant 1+\sum_{k=1}^{N} f_{k}+f_{N+1}+2 f_{N+2} .
$$

В теореме 2 возьмем $d=9, N=11, f_{1}=7.42800555, f_{2}=26.33181283, f_{3}=58.58939087$, $f_{4}=89.15779666, f_{5}=94.043798, f_{6}=65.60173825, f_{7}=23.05455021, f_{8}=f_{9}=0, f_{10}=$ $7.69458782, f_{11}=6.41689233, f_{12}=0.24656738, f_{13}=0.19223383$. Получим $\tau_{9} \leqslant 379.95$. Учитывая, что контактное число целое, имеем $\tau_{9} \leqslant 379$, что доказьвает теорему 1.

\section{СПИСОК ЛИТЕРАТУРЫ}

[1] Д. Конвей, Н. Слоэн. Упаковки шаров, решетки и группы. М.: Мир, 1990. [2] A. M. Odlyzko, N. J. A. Sloan // J. Combin. Theory Ser. A. 1979. V. 26. P. 210-214. [3] P. G. Boyvalenkov // Atti. Sem. Mat. Fis. Univ. Modena. 1994. V. 42. № 1. P. 159-163. [4] Г.А. Кабатянский, В.И. Левенштейн // Проблемы передачи информации. 1978. T. 14. № 1. C. $3-25$. 